\title{
Poligamia, matrimonio por el rito gitano y derecho a la pensión de viudedad: la reciente postura del Tribunal Constitucional frente al criterio \\ del Tribunal Supremo y su contradictorio impacto en la aplicación del derecho a la no discriminación
}

Polygamy, marriage by the gypsy rite and the right to a widow's pension: the recent position of the Constitutional Court against the criteria of the Supreme Court, and its contradictory impact on the application of the right to non-discrimination

\author{
Ma JOSÉ CERVILLA GARZÓN* \\ Profesora Titular de Derecho del Trabajo y de la Seguridad Social \\ Universidad de Cádiz (España) \\ mariajose.cervilla@uca.es
}

http://orcid.org/0000-0001-5245-3878

La presente reflexión trae causa de la inicial perplejidad que nos causa el diferente criterio con el cual los tribunales, Supremo y Constitucional, están valorando dos modelos de convivencia (pues, realmente, entendemos que como tales los podemos considerar, a falta de vínculos matrimoniales con validez en nuestro ordenamiento jurídico), en relación a sus efectos en el posible reconocimiento de pensiones de viudedad a alguno de los convivientes. Esta cuestión, que inicialmente podría considerarse fácil de resolver si nos atenemos al concluyente contenido de nuestra Ley General de Seguridad Social, sin embargo se torna mucho más compleja si lo ponemos en relación con su posible impacto en un derecho constitucional de transversal aplicación y sobre el cual nunca acaban los Tribunales de aclararse acerca de su interpretación y alcance: el derecho a la no discriminación. 
Poniendo el contenido de nuestras normas jurídicas en su contexto, el derecho a la pensión de viudedad se configura para un único beneficiario o beneficiaria, generalmente éste último caso, que ha contraído matrimonio o se ha constituido legalmente como pareja de hecho, con los condicionantes previstos en el art. 220 y ss de la Ley General de Seguridad Social. Pero obviando una visión simplista de la aplicación de la norma, que es lo que precisamente pretende evitar el reconocimiento constitucional del derecho a la no discriminación, sobre todo en su vertiente de prohibición de la discriminación indirecta, hay varias diversas circunstancias que concurren en nuestro entorno social y que no debemos pasar por alto. En primer lugar, que la pensión de viudedad tiene un destacado impacto de género, por cuanto sus beneficiarias son mayoritariamente mujeres (por citar un dato concreto, a diciembre de 2020, según los datos del Ministerio Inclusión, Seguridad Social y Migraciones, de 8.721 altas en la pensión de viudedad, sólo 1.003 corresponden a varones beneficiarios). En segundo lugar, que la sociedad española se caracteriza por una nada desdeñable diversidad de etnias, nacionalidades y religiones, algunas más presentes que otras, pero entre las que sin duda destacan los inmigrantes de origen marroquí, con la correlativa presencia de sujetos que profesan la religión islámica, y las personas que pertenecen a la etnia gitana. Quiere esto decir que hay que ser particularmente cuidadoso en la valoración de posibles aplicaciones de la norma que puedan implicar injustificadas desigualdades de trato por razón de religión, de nacionalidad o de origen étnico (o racial), pues la diversidad es nuestro sustrato social fundamental, preservándose, en paralelo, la protección de la mujer frente a la discriminación por razón de sexo.

En concreta relación a la incidencia de la población romaní en el globlal de la población española, siguiendo el estudio realizado en el año 2011 por el Ministerio de Sanidad, Política Social e Igualdad, "Diagnóstico social de la comunidad gitana en España", el 1,87\% de la población española pertenece a la etnia gitana, existiendo más de 7 millones en el contexto de la Unión Europea y siendo considerada como la primera minoría étnica europea. Otros informes incluso incrementan su presencia en el territorio español, avanzando la posible existencia de hasta 1.500.000 personas de esta etnia en el año 2019 ("Estudio comparado sobre la situación de la población gitana en España en relación al empleo y la pobreza", informe realizado por la Fundación Secretariado Gitano y el equipo ISEAK, Initiative for socio-economic analysis for knowledge). Este último también revela un dato preocupante sobre la posición económicolaboral de las mujeres en esta población, pues su tasa de actividad es muy inferior a la de los varones (un $38.5 \%$ ), incluso en comparación con la población general (donde las mujeres tienen una tasa de actividad del 53\%).

La doctrina judicial sobre la aplicación genérica del principio de igualdad y no discriminación está más que asentada, lo que no quiere decir que, ante distintas situaciones de posible discriminación basadas en causas diversas, se aplique con el mismo alcance y rigor. Resumiendo muy brevemente el contenido de esta doctrina, el principio de igualdad no implica que, en todos los casos, se ofrezca un tratamiento legal equivalente, de forma que no toda desigualdad de trato normativo respecto a la regulación de una determinada materia supone una infracción de este principio. Sólo se conculcará cuando se produzca una diferencia entre situaciones que se puedan considerar iguales, siempre que no exista una justificación objetiva 
y razonable. Para que la diferencia de trato sea lícita, desde la perspectiva constitucional, es imprescindible que las consecuencias jurídicas derivadas de la distinción sean proporcionadas a la finalidad perseguida, es decir, el juicio de proporcionalidad se realiza entre la medida adoptada, el resultado producido y la finalidad pretendida. Integrando tal principio el derecho a la no discriminación, éste implica la ilegitimidad de tratamientos diferenciados respecto de lo que operan como factores determinantes los enunciados por el art. $14 \mathrm{CE} \mathrm{u}$ otros, que pueden ser excepcionalmente utilizados como criterio de diferenciación jurídica siempre que superen el juicio de proporcionalidad de forma más estricta.

Sentadas todas estas premisas, emergen inevitables interrogantes: ¿está constitucionalmente justificado que se otorgue validez a una situación de poligamia para ser beneficiario de una pensión de viudedad, pero que no se reconozca la eficacia a los mismos efectos del matrimonio por el rito gitano?, ¿el impacto discriminatorio del no reconocimiento es mayor en un caso que en el otro, y por ello los Tribunales ofrecen soluciones diversas?. A bote pronto es evidente que la situación resulta muy poco comprensible si tenemos en cuenta que, por una parte, la poligamia es contraria al orden público español precisamente por su carácter discriminatorio, ofensivo y degradante para la mujer, e incluso es constitutiva de delito (no así una unión por el rito gitano), y, por otra, que es mucho más numerosa la población gitana que reside en el territorio español frente a posibles sujetos que profesen el islam, casados en situación de poligamia (sobre todo a raíz de la reforma de la Mudawana marroquí que mantiene la posible poligamia pero con mayores limitaciones), que puedan reunir los requisitos para solicitar la pensión del sistema español. Adicionalmente, el impacto discriminatorio de género puede ser mucho mayor en el caso de mujeres pertenecientes a la etnia gitana, puesto que la situación de doble discriminación en la que se pueden encontrar está, incluso, reconocida a nivel de la Unión Europea.

Pasemos, pues, a analizar las argumentaciones utilizadas por el Tribunal Supremo, en su sentencia de 17 de diciembre de 2019 (dictada en los mismos términos que su sentencia de 24 de enero de 2018), y por el Tribunal Constitucional, en su sentencia de 25 de enero de 2021 , para aclarar si la tutela antidiscriminatoria ha tenido algún impacto en sus resoluciones $\mathrm{y}$, de ser así, cual es el motivo que justifica lo contradictorio de sus pronunciamientos.

Por lo que se refiere al reconocimiento del derecho a la pensión de viudedad en caso de poligamia a todas las viudas, es cierto que hay un dato normativo que no se produce en el caso de los matrimonios celebrados por el rito gitano, y es que el Convenio hispano-marroquí sobre Seguridad Social contempla que todas las viudas pueden ser perceptoras de la pensión, excluyéndose a los trabajadores pertenecientes al Régimen de Clases Pasivas del Estado (art. 23). Pero la existencia de dicha norma no legitima, ni mucho menos, el reconocimiento del derecho a pensión si tenemos en cuenta, fundamentalmente, dos circunstancias. En primer lugar, que el convenio debe aplicarse de conformidad con el orden público español según en art. 12.3 Código Civil, en virtud del cual "en ningún caso tendrá aplicación la ley extranjera cuando resulte contraria al orden público", es decir, no puede incluir preceptos contrarios a los principios constitucionales. En segundo lugar, que, aun cuando demos por válida la aplicación de este convenio por entender que entra en juego la excepción del orden público atenuado, es 
decir, el hecho de otorgar efectos jurídicos "periféricos" a leyes extranjeras que, de suyo, atentan contra el orden público establecido, si no producen un daño sustancial a la sociedad, no perjudican a ningún particular y contribuyen a la instauración de la justicia formal, los Tribunales van mucho más allá porque ya se ha generalizado el reconocimiento del derecho a pensión de todas las viudas en los supuestos de poligamia, independientemente de su nacionalidad y de la posible aplicación o no del convenio citado.

Y ello teniendo en cuenta que los dos matrimonios no pueden ser válidos conforme a nuestro ordenamiento jurídico y, por lo tanto, no están ambos inscritos en el Registro Civil. De hecho, para poder formalizar la solicitud de la pensión, la entidad gestora requiere el Libro de familia, acta del Registro Civil o documento extranjero equivalente debidamente legalizado o sellado, en su caso, y traducido, que acredite el matrimonio con el causante fallecido. La legalización de estos matrimonios exige que el encargado del Registro Civil compruebe que la certificación matrimonial expedida en el extranjero se ajusta a la legalidad española y, como en el caso del matrimonio poligámico esto no es así, lo que impide su reconocimiento e inscripción, para que tales matrimonios puedan desplegar algún tipo de efecto es imprescindible que se admita el funcionamiento de la cláusula del orden público atenuado.

El reconocimiento del derecho a pensión sucede, en parte, por una argumentación que está vinculada al principio de no discriminación por razón de sexo, pero sin que podamos perder la perspectiva de que la poligamia es una situación ilegal según el código penal, y discriminatoria por denigrar la situación de la mujer conforme al art. 14 CE. Así, la sentencia del Tribunal Supremo de 17 de marzo de 2019 afirma que la determinación en singular de los beneficiarios de la pensión por la Ley General de Seguridad Social "ha de ser interpretado atemperándolo necesariamente a esos parámetros de igualdad, consagrados en el artículo 14 de la Constitución Española, en los casos en que nos encontremos con situaciones de poligamia", e, igualmente, que debe hacerse una interpretación "integradora e igualitaria" del artículo 23 del Convenio. Es más, según dicha sentencia, la aplicación del art. 14 es lo que legitima extender la previsión del contenido del convenio hispano-marroquí, por "vía interpretativa", a otras situaciones de poligamia no contempladas en dicho convenio.

$Y$ es que realmente la situación discriminatoria se puede producir desde la perspectiva del cónyuge casado que no va percibir pensión, en una aplicación estricta de las normas en juego, si tenemos en cuenta que es el varón el que puede decidir, en caso de reagrupamiento familiar, a cual de las cónyuges le causaría este perjuicio. Y esto, sin duda, supone un gravísimo atentado contra el principio de igualdad de trato y no discriminación constitucionalmente reconocido y que, por lo tanto, forma parte del orden público español. La justicia material se produce, tal y como afirma el Tribunal Supremo, con el reconocimiento de pensión a todas las viudas que, por otra parte, y esta cuestión parece que puede ser muy determinante, no produce ningún perjuicio económico al Sistema de Seguridad Social.

Pues bien, ¿Qué sucede en el caso del matrimonio por el rito gitano, siendo una situación conforme al orden público español, producida en una comunidad de gran impacto en el conjunto de la población española, que afecta a mujeres estadísticamente en situación de desigualdad en 
el acceso al empleo, para entender que no es aplicable el principio de no discriminación a los efectos de reconocerles el derecho a pensión de viudedad si no está inscrito civilmente el matrimonio?. En el caso de la poligamia, el Tribunal Supremo reinterpreta la exigencia de que el beneficiario de la pensión sea uno sólo en aras a la aplicación del principio de igualdad y no discriminación, por lo tanto, ¿no puede reinterpretarse la exigencia de unión matrimonial inscrita en el Registro Civil, o pareja de hecho constituida conforme a sus propias previsiones, en aplicación del mismo principio?.

En primer lugar, es muy importante considerar el contenido de resoluciones con posible incidencia en esta cuestión, dictadas con anterioridad a la sentencia del Tribunal Constitucional, si bien es cierto que ninguna responde, exactamente, a las mismas circunstancias. Aun así, de ellas es posible extraer algunas conclusiones.

La primera sentencia a destacar es la del Tribunal Constitucional de 15 de noviembre de 2004, en la cual afirmó la validez del matrimonio canónico, no inscrito en el Registro Civil, a efectos de considerar a los cónyuges sujetos causantes de pensión de viudedad al aplicar el art. 61 del Código Civil: "El matrimonio produce efectos civiles desde su celebración”. Pero, ciertamente, no podemos considerar que estemos ante circunstancias equivalentes con la aquí analizada, pues el matrimonio canónico se había celebrado de forma válida, pudiendo considerarse cónyuges legítimos al estar este matrimonio reconocido por nuestro ordenamiento jurídico ${ }^{1}$. No es lo que sucede con el matrimonio por el rito gitano, que no cuenta con ningún tipo de reconocimiento jurídico y, por ende, de validez.

De forma similar, pero en sentido contrario, el Tribunal Constitucional, en sentencia de 1 de diciembre de 2014, rechazó la validez de un matrimonio islámico, no inscrito en el Registro Civil, a efectos de causar los contrayentes derecho a pensión de viudedad, puesto que, en este caso, el matrimonio no era válido al no haberse presentado el preceptivo certificado de capacidad matrimonial y, por ello, no se podía inscribir.

Pero, sin duda, la sentencia más relevante para el caso que nos ocupa, como precedente a la sentencia del Tribunal Constitucional de 25 de enero de 2021, es la sentencia del Tribunal Europeo de Derechos Humanos, de 8 de diciembre de 2009 (asunto Muñoz Díaz contra España), que, aun cuando incorpora un elemento que falta en la sentencia que procedemos a comentar, integra algunas apreciaciones que, entendemos, deben tenerse muy en cuenta. Lo primero que debemos puntualizar es que esta sentencia determina que es válido un matrimonio celebrado por el rito gitano, a los efectos de ser sus contrayentes causantes del derecho a pensión de viudedad, pero no en base a la aplicación del principio de igualdad y no discriminación, sino por el hecho de que el Estado había admitido determinados actos jurídicos que podían llevar a los contrayentes a considerar, de buena fe, como válido su matrimonio (como determinados

\footnotetext{
${ }^{1}$ Conforme al art. 60.2 Código Civil: "se reconocen efectos civiles al matrimonio celebrado en la forma religiosa prevista por las iglesias, confesiones, comunidades religiosas o federaciones de las mismas que, inscritas en el Registro de Entidades Religiosas, hayan obtenido el reconocimiento de notorio arraigo en España".
} 
documentos de la Seguridad Social, donde aparecen como cónyuges, o en el propio libro de familia). Por lo tanto, sólo por lo concluyente de esta circunstancia, es decir, que el propio Estado estaba admitiendo una cierta legitimidad del matrimonio a través de actos muy concretos, ya era posible determinar que también fuese legítimo a efectos del percibo de pensiones de viudedad, sin tener que entrar en otras argumentaciones.

Sin embargo, lo destacable de esta sentencia, a nuestros efectos, es que el Tribunal adopta dos posturas ambivalentes, pero con una evidente intención de llamar la atención sobre la necesaria protección de la etnia gitana y su inevitable reconocimiento como población que necesita una tutela particular.

En primer lugar, realiza una serie de afirmaciones que, nos parece, suponen un cierto reconocimiento de la entidad que tiene el matrimonio celebrado por el rito gitano a efectos de la posible aplicación del principio de no discriminación por razón de pertenencia a una etnia determinada. En este sentido, llamamos la atención sobre el siguiente párrafo de la sentencia: "El Tribunal observa, a este respecto, que en el seno de los Estados contratantes del Consejo de Europa surge un consenso internacional para reconocer las necesidades particulares de las minorías y la obligación de proteger su seguridad, su identidad y su modo de vida (ver, apartado 33 supra, concretamente el Convenio-marco para la Protección de las Minorías no sólo con el objetivo de salvaguardar los intereses de las propias minorías sino también para preservar la diversidad cultural lo que beneficia a la sociedad en su conjunto. El Tribunal considera que, aunque la pertenencia a una minoría no dispensa de respetar las Leyes reguladoras del matrimonio, sí puede influir en la manera de aplicar estas Leyes. El Tribunal ha tenido ocasión en la sentencia Buckley (ciertamente, en un contexto diferente) de subrayar que la vulnerabilidad de la etnia gitana, por el hecho de constituir una minoría, implica prestar una especial atención a sus necesidades y a su modo de vida, tanto en el ámbito reglamentario admisible en materia de regulación como en el momento de la adopción de la decisión en casos particulares. Ciertamente, el artículo 174 de la Ley General de Seguridad vigente en el momento de los hechos, sólo reconocía la pensión de viudedad en caso de ausencia de matrimonio legal cuando el matrimonio era nulo de buena fe. Sin embargo, esta disposición no permite al Estado demandado exonerarse de toda responsabilidad en relación al Convenio".

Se deduce, sin duda, de su contenido, su reconocimiento de la etnia gitana como necesitada de una particular tutela, que debe influir en la manera de aplicar las leyes que afectan a la regulación del matrimonio, citando, en concreto, el Convenio-marco para la protección de las minorías. Incluso afirma, de forma expresa, que este matrimonio "no es contrario al orden público", para justificar el reconocimiento a la pensión. Como afirmábamos anteriormente, dado el reconocimiento implícito del matrimonio en determinados documentos oficiales estas afirmaciones tan categóricas casi eran innecesarias y por algún motivo estarán en el contenido de la sentencia, aun cuando su reflexión no se centra en la existencia de posible discriminación.

Pero, por otra parte, de forma bastante expresa rechaza que la legislación española esté vulnerando el principio de no discriminación al no admitir el matrimonio por el rito gitano a efectos de generar derecho a pensión, sobre lo que destacamos el siguiente párrafo: "Es cierto 
que algunas formas religiosas de consentimiento matrimonial están admitidas en la legislación española, pero estas formas religiosas están reconocidas en virtud de acuerdos anteriores de estas confesiones con el Estado y producen, por tanto, los mismos efectos que el matrimonio civil, mientras que otras formas (religiosas o tradicionales) no están reconocidas. Sin embargo, el Tribunal constata que se trata de una diferencia derivada de la pertenencia a una confesión religiosa, no aplicable en el caso de la comunidad gitana. Además, esta diferencia no impide o prohíbe el matrimonio civil, abierto a los gitanos en las mismas condiciones de igualdad que a las personas que no pertenecen a esta comunidad, y responde a motivos que el legislador debe tener en cuenta y que dependen, como subraya el Gobierno, de su margen de apreciación. Por tanto, el Tribunal considera que el hecho de que las uniones gitanas no originen efectos civiles en el sentido deseado por la demandante no constituye una discriminación ilegal considerando el artículo 14".

Entrando ya en el fondo de la sentencia del Tribunal Constitucional de 25 de enero de 2021, en la cual se demanda la validez del matrimonio por el rito gitano para generar derecho a pensión de viudedad, en base a la aplicación del principio de igualdad y no discriminación por razón de pertenencia a una determinada raza o etnia, en el supuesto de hecho planteado no concurren las circunstancias particulares que se daban al enjuiciar el asunto Muñoz Díaz contra España, al no haberse producido esa apariencia de legalidad en documentos oficiales. Para abundar en lo controvertido de este asunto, la sentencia cuenta con un Voto Particular, al igual que incluía uno la sentencia del Tribunal Supremo de 25 de enero de 2018, que se recurre en Amparo.

Dicha sentencia falló en contra de la validez del rito gitano a los efectos analizados, básicamente por razones parecidas a las esgrimidas por el Tribunal Europeo de Derechos Humanos: que el art. 174 de la Ley General de Seguridad Social (actuales arts. 200 y ss) es neutral, y que la vulnerabilidad de minorías étnicas o culturales no puede conllevar excepciones en la aplicación de la Ley so pena de comprometer la seguridad jurídica y la uniformidad normativa, haciéndose de peor condición a los que por razones ideológicas no se hayan constituido como pareja de hecho legal. Su Voto Particular, sin embargo, aprecia indicios de discriminación indirecta en la aplicación de los arts. 200 y ss. de la Ley General de Seguridad Social, aparentemente neutros, pues el colectivo de raza gitana se puede ver particularmente afectado debido a las particularidades de sus tradiciones. Por ello, entiende que una interpretación acorde al respecto de las minorías étnicas permitiría flexibilizar su aplicación si es posible afirmar que la convivencia se ha mantenido de forma real y efectiva, y con indiscutible carácter de pareja unida maritalmente.

Los razonamientos del Tribunal Constitucional, para mantener el criterio previo del Tribunal Supremo, se basan, fundamentalmente, en los siguientes argumentos:

- En primer lugar, que el art. $14 \mathrm{CE}$ no ampara la denominada "discriminación por indiferenciación", pues no consagra un derecho a la desigualdad de trato por no existir ningún derecho normativo a un trato desigual. No concurre, pues, una situación discriminatoria sino una decisión personal, libre y voluntaria de no acceder a las fórmulas de constitución en Derecho del vínculo matrimonial, las cuales no están condicionadas por la pertenencia a una 
raza y, por lo tanto, no es posible apreciar discriminación directa. De hecho, en palabras del Tribunal, a cualquier situación de no formalización de una relación de hecho se le denegaría también la pensión.

- En segundo lugar, y ya en relación a la posible apreciación de discriminación indirecta, el Tribunal realiza una afirmación que es el fundamental objeto de cuestionamiento por parte del Voto Particular. Así, teniendo en cuenta que la discriminación indirecta implica un tratamiento formalmente neutro del que, sin embargo, se deriva un impacto o resultado adverso sobre los miembros de un colectivo protegido, según el Tribunal no cabe advertir fundamento objetivo alguno de la existencia de un perjuicio mayor y verdaderamente desfavorable en la norma o en su aplicación, desde una perspectiva cualitativa o cuantitativa, en razón de pertenecer a esta minoría, frente al que puede acarrear para cualquier otro colectivo que opte por la no formalización de sus relaciones de convivencia.

Para afirmar la existencia de discriminación indirecta, el Voto Particular de la sentencia abunda en dos cuestiones, que sin duda pasa por alto el Tribunal Constitucional. La primera de ellas, al igual que apuntó el Tribunal Europeo de Derechos Humanos, se basa en el contenido del Convenio-Marco para la Protección de las Minorías Nacionales, cuyo art. 5 establece que es necesario promover las condiciones necesarias para permitir a las personas pertenecientes a minorías nacionales mantener y desarrollar su cultura, así como preservar los elementos esenciales de su identidad. Ciertamente a nuestro juicio no parece que la exigencia de inscripción registral del matrimonio sea una forma de no preservar un elemento esencial de la identidad propia de la etnia gitana, pero sí lo es si tenemos en cuenta la validez de otras fórmulas como la poligamia.

En segundo lugar, el Voto Particular, como es lógico, pone de manifiesto que, conforme a lo establecido en otras sentencias del Tribunal Constitucional, en la valoración de la discriminación indirecta debe atenderse a los datos por la estadística, y en este punto el impacto de la población romaní en el conjunto de la población española es más que evidente. Aporta, en este sentido, varios datos que sustentan el considerable impacto que, desde el punto de vista estadístico, tiene la denegación de los efectos del matrimonio por el rito gitano (suponen en torno al $1.57 \%$ de la población española, con un $28 \%$ de los miembros de su comunidad con personas casadas por su rito cultural tradicional, frente al $6 \%$ de la población general que está unida de hecho de manera informal, y con altos niveles de desempleo de larga duración). Incluso aprecia un sesgo de posible discriminación por motivo de género, atendiendo al dato anteriormente indicado de la gran prevalencia de mujeres beneficiarias de estas pensiones que, incluso, se puede incrementar en la población gitana, como apunta el Voto Particular, habida cuenta de que en el $83 \%$ de los casos el sustentando principal de la familia es el varón.

Como conclusiones para reflexionar sobre estas distintas valoraciones del alcance del principio de no discriminación, parece que la argumentación contenida en la sentencia del Tribunal Europeo de Derechos Humanos ciertamente nos llevaría a indicar que no hay discriminación directa en la denegación de efectos al matrimonio por el rito gitano al ser posible la inscripción registral, en igualdad de condiciones que en cualquier otro caso. Sin embargo, frente a este 
criterio, su propia llamada de atención a la necesaria protección de esta minoría, a la que obliga el Convenio-Marco para la Protección de las Minorías Nacionales, y los datos estadísticos puestos de manifiesto en el Voto Particular de la sentencia del Tribunal Constitucional, reveladores del impacto cualitativo y cuantitativo que tiene la formal neutralidad de las normas establecidas en la Ley General de Seguridad Social, dan, desde luego, para reflexionar sobre la necesidad de trascender su simple aplicación directa.

Pero cuando ponemos en conexión las argumentaciones del Tribunal Constitucional con el reconocimiento del derecho a pensión en situaciones de poligamia que valida el Tribunal Supremo, es cuando nos parece que se puede estar distorsionando la aplicación del derecho a la no discriminación, probablemente en función de un factor que, desgraciadamente, cada vez preside más la interpretación de las normas, cual es el impacto económico de las mismas. Basta con tener en cuenta las siguientes consideraciones:

- Tanto en el caso del matrimonio poligámico como en el celebrado por el rito gitano estamos ante matrimonios que no tienen legitimidad conforme a lo previsto en el Código Civil español. Pero hay que tener en cuenta una gran diferencia: que el celebrado por el rito gitano siempre será posible llegar a formalizarlo e inscribirlo, pero no así el poligámico al constituir esta situación un delito penal. Por lo tanto, cuanto el Tribunal afirma que a toda situación no legalmente formalizada se le denegará el derecho a pensión, eso no es cierto.

- Como expresamente afirmó el Tribunal Europeo de Derechos Humanos, el matrimonio celebrado por el rito gitano no es contrario al orden público y, sin embargo, si lo es la poligamia por discriminatoria por razón de género. Además, el Convenio de Seguridad Social sobre el que se apoya el reconocimiento del derecho a pensión es de carácter preconstitucional y, por lo tanto, podría ser considerado contrario al orden público.

- La poligamia no es una situación que ya tenga una gran incidencia en países islámicos, pero sí lo es la integración de la población gitana en el territorio español, es decir, en términos estadísticos sí tiene un impacto considerable la denegación de efectos al matrimonio por el rito gitano y, por ende, tiene impacto en una parte no despreciable de la población de sexo femenino. Por lo tanto, no cabe discusión sobre su estatus de minoría que necesita una especial protección y tutela.

- Por último, en el caso de la poligamia para reconocer el derecho a pensión se reinterpreta el contenido de un precepto que es claro, cual es el art. 219.1 de la Ley General de la Seguridad Social, al establecer que tiene derecho, en singular, el cónyuge superviviente. Sin embargo, la posible reinterpretación de otro precepto, cual es el art 221 de la Ley General de la Seguridad Social (al establecer las condiciones de las parejas de hecho), se rechaza para dar validez al matrimonio por el rito gitano, sin que exista una justificación objetiva y proporcionada.

Afirma el Tribunal Constitucional que "incluso cuando se ha optado por dotar de efectos en materia de prestaciones de seguridad social a otras formas de convivencia tampoco es posible apreciar connotaciones de exclusión étnica". Teniendo en cuenta lo expuesto, esta afirmación 
nos resulta particularmente controvertida, pues sí nos parece que hay una preferencia no justificada por la protección de la identidad islámica y las prácticas que les son propias, frente a la de una etnia que, conforme a sus propia identidad, cultura y valores, constituyen matrimonios válidos. Y tan legítimo es proteger la aplicación del principio de no discriminación por razón de sexo, en un caso, como el principio de no discriminación por razón de pertenencia a una etnia o raza en el otro, teniendo en cuenta, además, su tangencial impacto en la discriminación por razón de género.

Como razón última de este cuestionable tratamiento diverso de dos formas de convivencia frente a la tutela antidiscriminatoria, vislumbramos, como afirmamos con anterioridad, una motivación de carácter económico pues, si el reconocimiento del derecho a pensión a las distintas viudas en caso de poligamia sólo implica que una única pensión ya reconocida se va a repartir entre todas ellas, en caso de dar validez al matrimonio por el rito gitano se obliga al Sistema al desembolso económico que supone el pago de estas pensiones inicialmente no reconocidas. Lo que debemos plantearnos es si motivaciones vinculadas a la sostenibilidad del sistema de pensiones pueden ser las que deban determinar el mayor o menor alcance de la aplicación del principio de igualdad y no discriminación en nuestro ordenamiento jurídico. 